\title{
Doenças Crônico-Degenerativas em Adultos da Região Centro-Sul de Belo Horizonte: Análise sob a Perspectiva de Causas Múltiplas de Morte*
}

\author{
Chronic-Degenerative Diseases among Adults of the Center- \\ South Region of the Belo Horizonte City: Analyses by \\ Multiple Causes of Death Approach
}

\author{
Lenice Harumi Ishitani \\ Gerência de Regulação, Epidemiologia e Informação-GERSA-CS/Secretaria Municipal de Saúde de Belo Horizonte \\ Elisabeth França \\ Grupo de Estudos em Epidemiologia/DMPS/Faculdade de Medicina/UFMG
}

\begin{abstract}
Resumo
As estatísticas de mortalidade constituem um importante instrumento para a análise das condições de saúde das populações. Essa análise tem empregado, tradicionalmente, o conceito de causa básica de morte. Atualmente, entretanto, com o aumento da importância das doenças crônico-degenerativas como causas de morte, um maior número de diagnósticos tem sido informado nas declarações de óbito, dificultando a seleção de uma única causa de morte e levando a uma perda de informações relativas às afecções que não são selecionadas como causa básica de morte. Uma alternativa para isso é a utilização do enfoque de causas múltiplas de morte. Utilizou-se esse enfoque para estabelecer a magnitude da mortalidade de algumas doenças crônico-degenerativas na região Centro-Sul de Belo Horizonte. Foram analisados todos os óbitos ocorridos em adultos (20 a 69 anos de idade), no período de 1996 a 1998, por diabetes mellitus, doenças cerebrovasculares, doenças hipertensivas, fibrose e cirrose do fígado e doenças crônicas das vias aéreas inferiores. Os óbitos por diabetes mellitus e por doença hipertensiva, quando analisados sob o enfoque da causa básica de morte representaram 1,7 e 2,3\% do total, respectivamente, e, quando analisados através das causas múltiplas de morte, essas proporções aumentaram para 5,4 e 9,0\%. Considerando que as políticas de saúde devem propiciar a promoção e proteção da saúde, enfatizando o conhecimento e a prevenção dos fatores de risco, o enfoque das causas múltiplas de morte pode ser um importante instrumento no planejamento das ações de saúde para as doenças crônico-degenerativas.
\end{abstract}

\section{Palavras-Chave}

Mortalidade; Doenças Crônico-degenerativas; Causa da Morte; Causas Múltiplas de Morte.

\section{Summary}

Mortality statistics are an important tool to evaluate the population's health status. This analysis has traditionally been based on the concept of the underlying cause of death. Today, however, with the increasing proportion of death from chronic-degenerative diseases, an increased number of conditions are reported on death certificates, making assignment of a single cause difficult and resulting in the loss of information about conditions which are not classified as the underlying cause of death. Analysis based on multiple causes could be a new dimension in mortality statistics. This approach was used in this study of deaths caused by chronic diseases in the Center-South region of the city of Belo Horizonte. All deaths of adults aged 20 to 69 years which ocurred in the period between 1996 and 1998, caused by diabetes, cerebrovascular diseases, hypertension, cirrhosis and chronic lower respiratory tract diseases were considered. The proportional mortality of diabetes mellitus and hypertensive diseases was 1.7 and 2.3\%, respectively when analyzed based on the underlying cause of death, and rose to 5.4 and $9.0 \%$, respectively, when analyzed using the multiple cause of death approach. Health policies must provide health promotion and protection, with emphasis on knowledge and prevention of risk factors. The multiple causes of death approach could be an important instrument for planning and implementing effective health programs of chronic-degenerative diseases.

\section{Key Words}

Mortality; Chronic Diseases; Cause of Death; Multiple Causes of Death.

\footnotetext{
* Artigo originado de dissertação de Mestrado "Desigualdade Social e Causas Múltiplas de Morte em Adultos da Região Centro-Sul de Belo Horizonte”. Programa de Pós-graduação em Saúde Pública/Universidade Federal de Minas Gerais.

Endereço para correspondência: Gerência de Regulação, Epidemiologia e Informação/GERSA-CS/Secretaria Municipal de Saúde de Belo Horizonte - Rua Tupis, 149 - 7ํandar - Centro - Belo Horizonte/MG. CEP:30.130-007. E-mail:1enice.bhz@zaz.com.br
} 


\section{Introdução}

As estatísticas de mortalidade têm sido freqüentemente utilizadas para a análise das condições de saúde das populações. Entre as muitas variáveis de análise, como sexo, faixa etária, local de ocorrência e local de residência, a causa de morte constitui a mais importante. ${ }^{1,2}$

Tradicionalmente, emprega-se nas estatísticas de mortalidade o conceito de causa básica de morte por permitir maior comparabilidade entre diferentes locais, pela sua importância na análise de tendências históricas e por seu uso tradicional de indicar medidas de prevenção da morte. Entretanto, o enfoque da causa única adequava-se à descrição dos padrões de mortalidade do início do século passado, quando os óbitos eram devidos, em sua grande maioria, a "doenças agudas infecciosas" ou a violências. ${ }^{2,3}$

Com o envelhecimento da população e com o aumento da expectativa de vida, as doenças crônico-degenerativas tornaram-se importantes causas de morte. Devido ao curso prolongado dessas doenças e, muitas vezes, por apresentarem etiologia comum, observa-se um aumento do número de diagnósticos informados na declaração de óbito (DO), ${ }^{2,4}$ o que dificulta a seleção de uma única causa. Entre esses diagnósticos podem existir causas importantes de morte que não são classificadas como causa básica, não constando, portanto, nas estatísticas de mortalidade. Isso ocorre, por exemplo, com a hipertensão arterial e o diabetes, o que dificulta a avaliação dos programas de controle dessas doenças. O mesmo é observado em relação à prematuridade, também raramente classificada como causa básica. Diante desses fatos, ao selecionar apenas uma das patologias como causa de morte, perdem-se informações relativas aos demais diagnósticos contidos nas DO.

Outra limitação do enfoque da causa básica de morte é o preenchimento inadequado da $\mathrm{DO}$, por desconhecimento da maneira correta de fazê-lo ou devido à dificuldade do médico em selecionar a cadeia de acontecimentos que levou à morte. Nesse caso, ele pode deixar de informar as afecções concorrentes, não descrever o curso clínico completamente ou ainda, arbitrariamente, selecionar uma outra seqüência. ${ }^{5}$

Um outro aspecto importante é que, apesar de serem informadas corretamente como originárias, algumas afecções não são selecionadas como causa básica, em função das regras de seleção. ${ }^{1}$ Segundo a Décima Revisão da Classificação Estatística Internacional de Doenças e Problemas Relacionados à Saúde (CID10), em uma DO na qual a hipertensão foi a causa básica registrada e a doença isquêmica do coração, a causa associada, será considerada causa básica de morte a doença isquêmica. ${ }^{6}$

Falhas também podem ocorrer no momento da codificação das causas mencionadas na DO, decorrentes de erros de registro de código, desconsideração de algumas afecções mencionadas no atestado de óbito ou de aplicação incorreta de regras de codificação, alterando a seleção da causa básica de morte. ${ }^{2}$

Os outros diagnósticos, além da causa básica, que estavam presentes no momento da morte e que podem ter sido importantes na evolução para o óbito, devem ser conhecidos. Essas informações poderão ser obtidas através do estudo da mortalidade sob o enfoque de causas múltiplas de morte, o qual pode representar um importante subsídio para o planejamento das ações de saúde.

O estudo das causas múltiplas de morte tem sido discutido desde o início do século passado e foi utilizado para a análise de afecções que dificilmente seriam selecionadas como causa básica, tais como a hipertensão $\operatorname{arterial,}{ }^{7}$ o diabetes mellitus, ${ }^{8,9}$ a natureza das lesões em óbitos por causas externas. ${ }^{2,3}$ Também tem sido utilizado em estudos de alguns grupos ocupacionais, ${ }^{10} \mathrm{de}$ mortalidade infantil, ${ }^{11}$ estudos para a análise de agravos sob vigilância epidemiológica ${ }^{12}$ e em estudos de tendências da mortalidade.

Com o envelhecimento da população, os problemas de saúde do adulto tor- 
naram-se importantes. Apesar disso, a Saúde Pública no Brasil tem tradicionalmente priorizado ações voltadas para a criança, a mulher e o idoso, provocando um lapso em relação à assistência ao adulto - o período de vida mais produtivo de uma pessoa. ${ }^{13}$ Assim, conhecer a mortalidade dessa faixa etária torna-se fundamental para a avaliação da situação de saúde e o planejamento dos serviços de saúde.

Diante dessas considerações e visando quantificar a mortalidade por algumas doenças crônicas não transmissíveis bem como identificar a associação das causas informadas nas declarações de óbito, propôs-se utilizar o enfoque das causas múltiplas de morte para análise dos óbitos de adultos por diabetes mellitus, doenças cerebrovasculares, doenças hipertensivas, fibrose e cirrose do fígado e doenças crônicas das vias aéreas inferiores (bronquites, enfisema, asma e doenças pulmonares obstrutivas crônicas).

\section{Material e métodos}

Foram utilizadas as informações referentes aos óbitos de 1996 a 1998, de adultos na faixa etária de 20 a 69 anos, residentes na regional Centro-Sul de Belo Horizonte, com uma população estimada em 250 mil habitantes.

Os dados contidos na base de dados do Sistema de Informações sobre Mortalidade (SIM) - Ministério da Saúde foram processados no Núcleo de Epidemiologia do Distrito Sanitário Centro-Sul (DISACS). Esses dados foram complementados com os obtidos por busca ativa, na Fundação João Pinheiro, das DO que não constavam na base de dados do SIM do DISACS.

As causas de óbito informadas na declaração de óbito foram codificadas, segundo CID-10. ${ }^{14}$ Em se tratando de estudo sob a perspectiva das causas múltiplas de morte, é necessária a informação precisa sobre o número de vezes que cada diagnóstico foi mencionado. Dessa forma, nas DO em que houve codificação da associação de causas, cada afecção foi, posteriormente, codificada individualmente.

Após a codificação, todas as causas mencionadas de óbito foram digitadas no programa Sistema de Seleção de Causa Básica (SCB-10 - Décima Revisão), ${ }^{15}$ programa compatível com o SIM e que procede à seleção da causa básica de óbito, padronizando a utilização das regras de classificação das causas, segundo a CID-10. ${ }^{6}$

Os diagnósticos mencionados nas DO foram agrupados segundo a Lista Brasileira para Mortalidade da CID-10 (CID-BR). ${ }^{16}$ A CID-BR, elaborada em 1980 pela Divisão Nacional de Epidemiologia e Centro Brasileiro de Classificação de Doenças (CBCD), é uma lista resumida que leva em conta as causas de óbito mais relevantes no quadro nosológico brasileiro, permitindo a padronização da apresentação dos dados, e é atualizada conforme revisão da CID.

As causas de óbito foram analisadas segundo o conceito de causa básica e causas múltiplas de morte. A definição da causa básica de morte utilizada foi aquela padronizada em 1948, quando foi aprovada a Sexta Revisão da CID, ou seja: "(a) a doença ou lesão que iniciou a cadeia de acontecimentos patológicos que conduziram diretamente à morte ou (b) as circunstâncias do acidente ou violência que produziram a lesão fatal". ${ }^{6}$ Causas múltiplas são aquelas referentes à classificação de todas as causas básicas e associadas - mencionadas nos atestados. As causas associadas são constituídas pelas causas conseqüenciais e as contribuintes. As conseqüenciais são aquelas que sucedem a causa básica. Causas contribuintes são aquelas que não fazem parte da cadeia iniciada pela básica, e que, no atestado, são declaradas na Parte II. ${ }^{1}$

Para obtenção das informações sobre a causa básica e as causas múltiplas de morte, foi utilizado o Tabulador de Causas Múltiplas de Morte (TCM), programa específico e compatível com o SIM e o SCB-10. Esse programa constrói uma tabela matriz que oferece a freqüência das causas mencionadas nas 
Tabela 1 - Distribuição de óbitos de adultos e total de diagnósticos*, segundo o número de diagnósticos mencionados por declaração de óbito (DO) na região Centro-Sul de Belo Horizonte - 1996 a 1998

\begin{tabular}{lrrrrr}
\hline $\begin{array}{l}\text { Número de } \\
\text { diagnósticos }\end{array}$ & \multicolumn{2}{c}{ Óbitos } & & \multicolumn{2}{c}{ Total de diagnósticos } \\
\cline { 2 - 3 } & $\mathbf{N}^{\mathbf{0}}$ & $\mathbf{\%}$ & & $\mathbf{N}^{0}$ & $\mathbf{\%}$ \\
\hline 1 & 364 & 16,3 & & 364 & 5,9 \\
2 & 610 & 27,2 & & 1.220 & 19,8 \\
3 & 742 & 33,1 & & 2.226 & 36,1 \\
4 & 331 & 14,8 & & 1.324 & 21,5 \\
5 & 131 & 5,9 & & 655 & 10,6 \\
6 & 48 & 2,1 & & 288 & 4,7 \\
7 & 13 & 0,6 & & 91 & 1,5 \\
Total & $\mathbf{2 . 2 3 9}$ & $\mathbf{1 0 0 , 0}$ & & $\mathbf{6 . 1 6 8}$ & $\mathbf{1 0 0 , 0}$ \\
\hline
\end{tabular}

Fonte: Sistema de Informações sobre Mortalidade (SIM) / Núcleo de Epidemiologia / Distrito Sanitário Centro-Sul (DISACS)

* Média de diagnósticos por $\mathrm{DO}=2,76$ e desvio-padrão=1,24.

DO, o número e a média de diagnósticos mencionados na DO segundo a causa básica de morte. Além disso, fornece a combinação dessas causas e o cálculo da razão entre o total de menções de uma determinada causa e o número de vezes em que foi selecionada como básica (razão menções/causa básica). Na análise das causas múltiplas de morte é necessária a eliminação das multiplicações de diagnósticos, realizadas pelo TCM. Esta multiplicação de diagnósticos ocorre quando as causas múltiplas são tabuladas em graus de agregação maiores do que aqueles em que os dados foram produzidos, ou seja, quando se aumenta o grau de agregação o número de causas associadas tende a diminuir. ${ }^{17}$ Sem essa correção, ocorreria a desnecessária verificação de associação de uma doença com ela mesma. ${ }^{2}$

Selecionou-se para estudo os seguintes grupos de causas e respectivos códigos da CID-10: diabetes mellitus (E10 - E14), doenças hipertensivas (I10 - I13), doenças cerebrovasculares (I60 - I69), fibrose e cirrose do fígado (K74) e o grupo das doenças crônicas das vias aéreas inferiores (bronquites, enfisema, asma, doenças pulmonares obstrutivas crônicas, identificadas no grupo da CID10 pelos códigos J40 - J47).

\section{Resultados}

Em 2.239 DO de adultos residentes na região Centro-Sul, ocorridos no período de 1996 a 1998, foram mencionados 6.168 diagnósticos, resultando em uma média de 2,76 diagnósticos informados por DO (desvio-padrão $=1,24)($ Tabela 1$)$. Observa-se que, em apenas $16,3 \%$ das DO, estava informada somente uma causa de morte e que, pelo enfoque da causa básica de morte, se deixariam de estudar as informações relativas aos outros diagnósticos mencionados em $83,7 \%$ das DO.

As causas básicas mais importantes de morte neste período foram as doenças do aparelho circulatório, as neoplasias e as causas externas, que corresponderam a $67,8 \%$ dos óbitos (Tabela 2). As doenças isquêmicas do coração ( $\mathrm{n}=198)$, a doença hipertensiva $(\mathrm{n}=52)$ e as doenças cerebrovasculares $(\mathrm{n}=160)$ foram responsáveis por $62,9 \%$ dos óbitos por doenças do aparelho circulatório. As mortes violentas como homicídios $(\mathrm{n}=139)$ e acidentes de transporte $(n=122)$, corresponderam a um total de $70 \%$ dos óbitos por causas externas.

Ao analisar as causas de morte sob a perspectiva das causas múltiplas, observa-se um aumento da importância relativa de quase todas as causas de morte evidenciada pela razão menções/causa básica maior que um (Tabela 2). Esse aumento, que atingiu $525 \%$ nas doenças do sangue e dos órgãos hematopoiéticos, só não foi maior que $50 \%$ (razão menções/causa básica maior ou igual a $1,50)$ nas neoplasias, nas doenças cardiovasculares, nas complicações da gravidez, parto e puerpério e nas causas externas. Essas causas foram também as que apresentaram menores médias de diagnósticos por DO. Observa-se que a média apresentada na Tabela $2(2,13)$ é menor do que a apresentada na Tabela 1 $(2,76)$. Tal fato deve-se à eliminação da multiplicação de diagnósticos decorrente 
Tabela 2 - Óbitos e mortalidade proporcional de adultos segundo causa básica e causas múltiplas de morte, agrupadas por capítulos da Classificação Internacional de Doenças (CID-10) na região Centro-Sul de Belo Horizonte - 1996 a 1998

\begin{tabular}{|c|c|c|c|c|c|c|}
\hline \multirow{2}{*}{ Causas de morte } & \multicolumn{2}{|c|}{ Causa básica } & \multicolumn{2}{|c|}{ Causa mencionada } & \multirow{2}{*}{$\begin{array}{l}\text { Razão } \\
(\mathrm{M} / \mathrm{B})^{* *}\end{array}$} & \multirow{2}{*}{ Média**** } \\
\hline & $\mathbf{N}^{\circ}$ & $\% *$ & $\mathbf{N}^{0}$ & $\% *$ & & \\
\hline Doenças infecciosas e parasitárias & 138 & 6,2 & 336 & 15,0 & 2,43 & 2,46 \\
\hline Neoplasias & 492 & 22,0 & 526 & 23,5 & 1,07 & 2,07 \\
\hline Doenças do sangue e hematopoéticas & 8 & 0,4 & 50 & 2,2 & 6,25 & 2,88 \\
\hline Doenças endócrinas, metababolismo e nutricionais & 47 & 2,1 & 232 & 10,4 & 4,94 & 3,06 \\
\hline Transtornos mentais e comportamentais & 25 & 1,1 & 88 & 3,9 & 3,52 & 2,60 \\
\hline Doenças do sistema nervoso & 45 & 2,0 & 104 & 4,6 & 2,31 & 2,71 \\
\hline Doenças do olho e anexos & - & - & 1 & 0,0 & - & - \\
\hline Doenças do aparelho circulatório & 652 & 29,1 & 888 & 39,7 & 1,36 & 1,97 \\
\hline Doenças do aparelho respiratório & 178 & 7,9 & 707 & 31,6 & 3,97 & 2,19 \\
\hline Doenças do aparelho digestivo & 142 & 6,3 & 255 & 11,4 & 1,80 & 2,38 \\
\hline Doenças da pele, tecido subcutâneo & 9 & 0,4 & 15 & 0,7 & 1,67 & 3,89 \\
\hline Doenças osteomusculares, tecido conjuntivo & 6 & 0,3 & 18 & 0,8 & 3,00 & 3,67 \\
\hline Doenças do aparelho geniturinário & 36 & 1,6 & 138 & 6,2 & 3,83 & 2,97 \\
\hline Gravidez, parto e puerpério & 2 & 0,1 & 2 & 0,1 & 1,00 & 1,50 \\
\hline Malformações congênitas & 6 & 0,3 & 9 & 0,4 & 1,50 & 3,33 \\
\hline Mal definidas & 80 & 3,6 & 680 & 30,4 & 8,50 & 3,33 \\
\hline Lesões e envenenamentos & - & - & 253 & 11,3 & - & - \\
\hline Causas externas & 373 & 16,7 & 452 & 20,2 & 1,21 & 2,02 \\
\hline Fat. influenc. saúde e cont. serviços**** & - & - & 5 & 0,2 & - & \\
\hline Total de óbitos & 2.239 & 100,0 & 4.759 & Não-calculada & 2,13 & 2,13 \\
\hline
\end{tabular}

Fonte: Sistema de Informações sobre Mortalidade (SIM) / Núcleo de Epidemiologia / Distrito Sanitário Centro-Sul (DISACS).

* $\quad$ Percentual em relação ao total de óbitos.

** $\quad$ Razão $(\mathrm{M} / \mathrm{B})$ = razão causa mencionada / causa básica.

*** Média de diagnósticos por declaração de óbito, segundo a causa básica de morte (agrupadas segundo capítulos da CID-10), excluída a multiplicação de causas.

**** Fatores que influenciam o estado de saúde e o contato com os serviços de saúde.

do maior grau de agregação dos agrupamentos, conforme capítulos da CID10.

A Tabela 3 apresenta algumas doenças crônicas não transmissíveis como causas de morte e observa-se que nas $542 \mathrm{DO}$ em que foram selecionadas como causa básica de morte, foram mencionadas em média mais de 2,54 diagnósticos por DO, atingindo uma média de 3,35 no diabetes mellitus.

Observa-se que, na região CentroSul, alguns diagnósticos como o diabetes mellitus e a doença hipertensiva foram mencionados pelo menos duas e três vezes mais do que selecionados como causa básica (razão menções/causa básica de 3,30 e 3,88, respectivamente). No caso do diabetes mellitus, quando analisado sob o enfoque da causa básica de morte, a proporção de óbitos foi de
$1,7 \%$ e, quando analisado através das causas múltiplas de morte, esta proporção aumentou para 5,4\%. Na doença hipertensiva, passou de 2,3 para $9 \%$. Com a utilização das causas múltiplas de morte, ocorreu também o aumento das outras doenças crônicas não transmissíveis referidas na Tabela 3.

No estudo de associação de causas (Tabela 4), verifica-se que, quando o diabetes mellitus foi selecionado como a causa básica de morte, as doenças hipertensivas apareceram como causa associada em 29,7\% $(n=11)$ das DO, enquanto a septicemia, as doenças isquêmicas do coração e as doenças cerebrovasculares em $18,9 \%(n=7)$ e a insuficiência renal em 21,6\% $(n=8)$. As doenças hipertensivas, por sua vez, foram mencionadas como causa associada em $33,8 \%(n=54)$ e em 20,7\% ( $n=41)$, 
Tabela 3 - Óbitos e mortalidade proporcional de adultos segundo causa básica e causas múltiplas de morte, e razão (M/B), por algumas causas de morte na região Centro-Sul de Belo Horizonte - 1996 a 1998

\begin{tabular}{|c|c|c|c|c|c|c|}
\hline \multirow{2}{*}{ Causas de morte } & \multicolumn{2}{|c|}{ Causa básica } & \multicolumn{2}{|c|}{ Causa mencionada } & \multirow{2}{*}{$\begin{array}{c}\text { Razão } \\
(\mathrm{M} / \mathrm{B})(\mathbf{b} / \mathbf{a}) * *\end{array}$} & \multirow{2}{*}{ Média*** } \\
\hline & $\mathrm{N}^{0}$ (a) & $\%$ & $\mathbf{N}^{0}(\mathbf{b})$ & $\% *$ & & \\
\hline Diabetes mellitus & 37 & 1,7 & 122 & 5,4 & 3,30 & 3,35 \\
\hline Doença hipertensiva & 52 & 2,3 & 202 & 9,0 & 3,88 & 2,44 \\
\hline Doença cerebrovascular & 160 & 7,1 & 225 & 10,0 & 1,41 & 2,45 \\
\hline Doenças isquêmicas do coração & 198 & 8,8 & 244 & 10,9 & 1,23 & 2,42 \\
\hline Doenças crônicas de vias aéreas inferiores & 43 & 1,9 & 68 & 3,0 & 1,58 & 2,91 \\
\hline Fibrose e cirrose do fígado & 52 & 2,3 & 91 & 4,1 & 1,75 & 2,50 \\
\hline Total & 542 & 24,2 & 952 & 42,5 & 1,76 & 2,54 \\
\hline
\end{tabular}

Fonte: Sistema de Informações sobre Mortalidade (SIM) / Núcleo de Epidemiologia / Distrito Sanitário Centro-Sul (DISACS).

* Percentual em relação ao total de óbitos.

* Razão causa mencionada / causa básica.

*** Média de diagnósticos por declaração de óbito, excluída a multiplicação de diagnósticos.

Tabela 4 - Distribuição percentual* dos principais grupos de causas associados em que diabetes mellitus, doença hipertensiva, doença cérebrovascular, doenças crônicas das vias aéreas inferiores, fibrose e cirrose do fígado e doenças isquêmicas do coração foram causa básica na região Centro-Sul de Belo Horizonte - 1996 a 1998

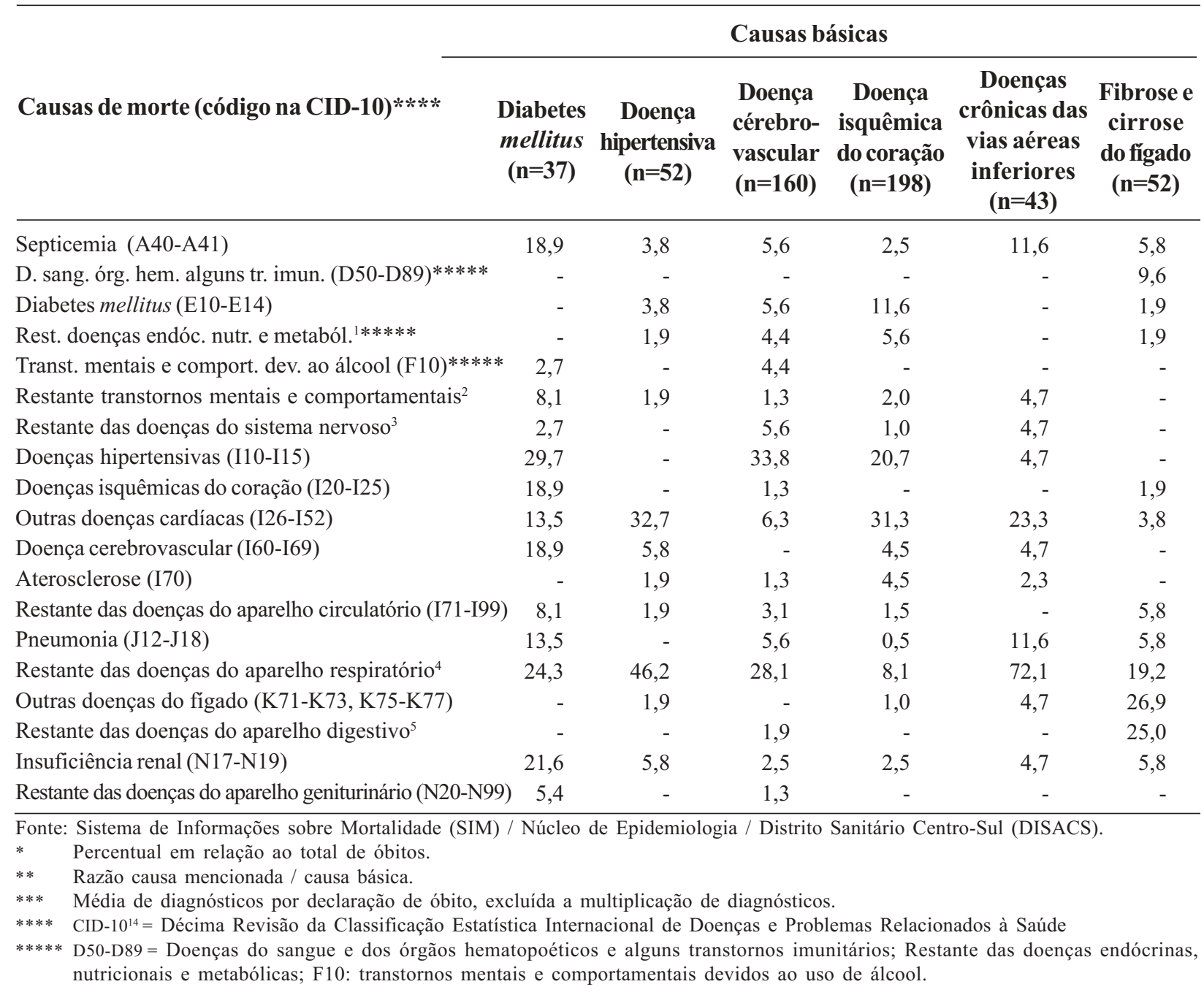


respectivamente, das DO cujas causas básicas foram as doenças cerebrovasculares e as doenças isquêmicas do coração.

Quando o diabetes mellitus foi causa associada (Tabela 5), observa-se novamente a relação com as doenças isquêmicas do coração que foram a causa básica em 27,1\% $(n=23)$. A doença hipertensiva foi causa associada em $27,3 \%(n=41)$ e $36,0 \%(n=54)$ das vezes em que as doenças isquêmicas do coração e as doenças cerebrovasculares foram, respec-tivamente, selecionadas como causas básicas de morte. Encontrou-se também uma relação entre fibrose e cirrose do fígado com hepatite viral e neoplasia de fígado e vias biliares (10,3\%). Em relação às doenças crônicas das vias aéreas inferiores, observa-se uma maior importância das doenças do aparelho respiratório e das cardiovasculares.

\section{Discussão}

A análise da mortalidade de adultos é relevante, pois permite identificar importante parcela de mortes prematuras, tendo em vista que a esperança média de vida do brasileiro aproxima-se dos 70 anos de idade..$^{18}$ Essas mortes prematuras representam um ônus para a sociedade, pois indicam redução de vida produtiva e perda

Tabela 5 - Distribuição percentual* das principais causas básicas de óbitos em que diabetes mellitus, doença hipertensiva, doença cerebrovascular, doenças crônicas das vias aéreas inferiores, fibrose e cirrose do fígado e doenças isquêmicas do coração foram causas associadas na região Centro-Sul de Belo Horizonte - 1996 a 1998

\begin{tabular}{|c|c|c|c|c|c|c|}
\hline \multirow[b]{2}{*}{ Causas de morte (código na CID-10***) } & \multicolumn{6}{|c|}{ Causas associadas } \\
\hline & $\begin{array}{l}\text { Diabetes } \\
\text { mellitus } \\
(\mathrm{n}=35) * *\end{array}$ & $\begin{array}{c}\text { Doença } \\
\text { hipertensiva } \\
(\mathbf{n = 1 5 0} * *\end{array}$ & $\begin{array}{l}\text { Doença } \\
\text { cérebro- } \\
\text { vascular } \\
(n=65) * *\end{array}$ & $\begin{array}{c}\text { Doença } \\
\text { isquêmica } \\
\text { do coração } \\
(n=46) * *\end{array}$ & $\begin{array}{c}\text { Doenças } \\
\text { crônicas das } \\
\text { vias aéreas } \\
\text { inferiores } \\
(\mathbf{n}=\mathbf{2 5})^{* *}\end{array}$ & $\begin{array}{l}\text { Fibrose e } \\
\text { cirrose } \\
\text { do fígado } \\
(n=39) * *\end{array}$ \\
\hline Hepatite viral (B15-B19) & 1,2 & - & 1,5 & - & - & 10,3 \\
\hline Neoplasia maligna do fígado e vias biliares (C22) & - & - & - & - & - & 10,3 \\
\hline Diabetes mellitus (E10-E14) & - & 7,3 & 10,8 & 15,2 & - & - \\
\hline Rest. doenças endóc. nutr. e metaból. ${ }^{1 * * * *}$ & 1,2 & 2,0 & 1,5 & 6,5 & - & - \\
\hline Doenças hipertensivas (I10-I15) & 2,4 & - & 4,6 & - & 4,0 & 5,1 \\
\hline Doenças isquêmicas do coração (I20-I25) & 27,1 & 27,3 & 13,8 & - & 12,0 & 2,6 \\
\hline Outras doenças cardíacas (I26-I52) & 12,9 & 4,0 & 13,8 & 13,0 & 24,0 & 7,7 \\
\hline Doença cerebrovascular (I60-I69) & 10,6 & 36,0 & - & 4,3 & 8,0 & - \\
\hline Restante das doenças do aparelho circulatório (I71-I99) & 9) 1,2 & 4,0 & 3,1 & - & 12,0 & - \\
\hline Pneumonia (J12-J18) & 5,9 & 3,3 & 3,1 & 10,9 & 4,0 & 5,1 \\
\hline Restante das doenças do aparelho respiratório ${ }^{2}$ & 4,7 & - & 3,1 & 6,5 & 8,0 & 5,1 \\
\hline Outras doenças do fígado (K71-K73, K75-K77) & - & 0,7 & - & - & 4,0 & 28,2 \\
\hline Restante das doenças do aparelho digestivo ${ }^{3}$ & - & 2,0 & - & 6,5 & - & 12,8 \\
\hline Restante das doenças do aparelho geniturinário (N20-N99) & 9) 3,5 & 0,7 & 1,5 & 4,3 & - & - \\
\hline
\end{tabular}

Fonte: Sistema de Informações sobre Mortalidade (SIM) / Núcleo de Epidemiologia / Distrito Sanitário Centro-Sul (DISACS)

* Percentual em relação ao total de menções como causas associadas.

** Menções como causa associada.

*** CID-10 ${ }^{14}$ Décima Revisão da Classificação Estatística Internacional de Doenças e Problemas Relacionados à Saúde

**** Restante das doenças endócrinas, nutricionais e metabólicas;

$=$ zero.

(E00-E-07, E15-E35, E50-E90)

(J00-J06, J30-J39, J60-J98)

(K00-K22, K28-K64, K66, K80, K82-K93) 
da qualidade de vida da família, provocando impactos social e econômico negativos. ${ }^{19}$

Para utilização e interpretação adequada das causas múltiplas de morte, é necessário estar ciente da principal limitação desse método de análise - a dependência da magnitude com que os médicos relatam as causas associadas. Assim como ocorre com o enfoque da causa básica de morte, as estatísticas de mortalidade por causas múltiplas resultam da opinião médica, que pode variar segundo a escola e a área geográfica em que o médico atua ou segundo a compreensão de qual causa deverá ser incluída na declaração de óbito. ${ }^{3}$

Apesar dessas limitações, os resultados deste estudo mostraram que a mortalidade por algumas doenças crônico-degenerativas podem ser subestimadas, tanto em decorrência da evolução crônica das doenças quanto dos procedimentos internacionais de seleção da causa básica de morte adotados pela Organização Mundial da Saúde (OMS). ${ }^{9}$

Optou-se por trabalhar apenas com uma região e não com todo o município, com base na proposta de planejamento e atuação descentralizada da Prefeitura de Belo Horizonte e da impossibilidade do reprocessamento, em tempo hábil, de aproximadamente 4 mil DO por ano da base de dados da Fundação João Pinheiro, nos quais foram utilizados códigos de associação. Neste caso, no processo de codificação, outras causas mencionadas podem não ter sido devidamente codificadas e, portanto, não digitadas, o que poderia distorcer a interpretação dos resultados encontrados pela metodologia das causas múltiplas de morte. Seriam, então, necessários o resgate dessas DO, a conferência da codificação, a recodificação e posterior digitação. Foi necessário, então, o estudo num período de três anos (1996 a 1998) para analisar a associação de causas, pois o número de óbitos ocorrido durante o período de um ano foi insuficiente para tal análise.

A proporção de DO que apresentava apenas uma causa mencionada foi menor que a encontrada por Laurenti, ${ }^{1}$ Israel $^{3} \mathrm{e}$ Santo, ${ }^{2}$ o que pode sugerir tendência de aumento do número de diagnósticos informados por DO. O número médio de causas informadas nas DO foi semelhante ao encontrado no Estado de São Paulo. ${ }^{17}$ Essa informação, relacionada à qualidade do preenchimento da DO e à assistência prestada ao falecido, ${ }^{11,17}$ pode ser um importante indicador da qualidade dos dados utilizados para este estudo. Ressalta-se que, quanto maior for a proporção de DO com mais de um diagnóstico, maior é a perda de informação sobre as causas de morte, quando analisadas sob o enfoque da causa única. ${ }^{11}$ As causas, cujas DO apresentaram médias de número de diagnósticos me-nores podem ser conseqüência do fato de outras patologias presentes no momento do óbito não serem mencionadas em função do diagnóstico bem determinado como no caso de causas externas e neoplasias; ou, ao contrário, serem referentes aos óbitos sem diagnósticos definidos ou relativas àquelas DO com preenchimento de afecções terminais de doenças.

Com o enfoque das causas múltiplas de morte, evidenciou-se que o diabetes mellitus e a doença hipertensiva são subestimadas quando analisadas sob a perspectiva da causa básica de morte (razão menções/causa básica maior que 3). A proporção de óbitos por diabetes mellitus encontrada neste estudo $(1,7 \%)$ foi menor que a apresentada por Franco e colaboradores, ${ }^{8}$ em 1992, no Estado de São Paulo $(2,7 \%)$. Isto pode ser explicado pela faixa etária da população deste estudo estar restrita de 20 a 69 anos. A mortalidade proporcional por diabetes mellitus, quando analisada pela perspectiva das causas múltiplas de morte em relação ao enfoque da causa básica de morte, apresentou, um incremento de $230 \%$, maior que os $140 \%$ relatado em Recife $^{9}$ e maior que a encontrada por Franco e colaboradores $^{8}(160 \%)$ no Estado de São Paulo. Esse aumento pode ser conseqüência da evolução crônica da doença, do aumento da esperança de vida e de melhoria da assistência à saúde. Assim, os diabéticos passam a morrer menos da doença e mais de suas com- 
plicações crônicas, por exemplo as cardiovasculares, que passam a figurar como causas básicas de morte subestimando o diabetes. Este aspecto ressalta a necessidade de se empregar nos estudos de mortalidade, a análise das causas múltiplas, quando se deseja conhecer a real importância do diabetes como causa de morte. Essa abordagem também permite conhecer o perfil de morbidade associada ao diabetes por ocasião do óbito. As doenças cardiovasculares tais como as isquêmicas do coração, as cerebrovasculares e as hipertensivas mostraram-se importantes como já citado por outros autores. ${ }^{8,9}$ Esse achado pode ser relacionado a resultados encontrados em outros estudos, que comprovaram um risco maior de morte de pacientes diabéticos por doenças cardiovasculares, entre esses a doença cerebrovascular, além de ser também uma causa importante de insuficiência renal. ${ }^{8,20}$

Conhecer a magnitude do diabetes mellitus na mortalidade é fundamental por ser prevalente em adultos; por apresentar graves complicações e incapacidades precoces; por ser fator de risco cardiovascular; e por ser uma enfermidade que gera encargos, tanto para os pacientes como para a sociedade. Constitui agravante para a Saúde Pública o ônus gerado pelo aumento da prevalência do diabetes mellitus decorrente da tecnologia médica e do aumento da expectativa de vida. Assim, a metodologia das causas múltiplas de morte poderá ser útil para o planejamento da prevenção primária e da atenção ao paciente diabético que deverá incluir não só o diagnóstico e o tratamento, mas, também a reabilitação social e profissional e a educação sobre a enfermidade. ${ }^{20}$

Observou-se que a hipertensão arterial foi mencionada três vezes mais do que foi selecionada como causa básica, apresentando uma razão menções/causa básica menor que a registrada para o Estado de São Paulo. ${ }^{17}$ Essa razão maior que três é decorrente de uma regra de seleção que dá preferência às outras causas (por exemplo doença cerebro- vascular e doenças isquêmicas do coração). A maior proporção de causas associadas às doenças hipertensivas, quando esta foi causa básica, foram relativas às doenças cardíacas, seguidas das doenças restantes do aparelho respiratório. Evidenciou-se também a importância da insuficiência renal e das doenças cerebrovasculares. Quando as doenças hipertensivas foram causas associadas de morte, as principais causas básicas foram as doenças isquêmicas do coração e as doenças cerebrovasculares. Esses resultados foram semelhantes aos encontrados por Santo. ${ }^{2}$

A análise da mortalidade através da perspectiva das causas múltiplas de morte evidenciou que as doenças cerebrovasculares são mencionadas $40 \%$ mais do que são selecionadas como causa básica de morte, demonstrando que essas doenças são ainda mais freqüentes do que o divulgado nas estatísticas definidas pela causa única. A razão de menções/causa básica foi semelhante à encontrada por Santo e Pinheiro. ${ }^{17}$ Em relação à associação das causas de morte, Santo ${ }^{2}$ já tinha apontado a relevância das doenças hipertensivas e das demais doenças respiratórias como causas associadas de morte. Quanto às causas básicas mais freqüentes, essas também foram as doenças isquêmicas do coração, outras doenças cardíacas e o diabetes mellitus. Considerando que as doenças cerebrovasculares estão associadas à hipertensão arterial, fica evidente a necessidade do programa de controle da hipertensão arterial. Constituem fatores que contribuiriam para a redução da mortalidade por hipertensão arterial: o aumento da conscientização pública quanto aos fatores de risco cardio e cerebrovasculares; as mudanças significativas nos estilos de vida, como o aumento da atividade física e a diminuição do hábito de fumar; o desenvolvimento de terapia efetiva para hipertensão; e o acesso à assistência. Com efetivos programas de controle da hipertensão, seria possível uma redução considerável do número de mortes precoces por complicações dessa doença e da doença cerebrovascular. ${ }^{7,19}$ 
Em relação às doenças crônicas das vias aéreas inferiores, observou-se que com o enfoque das causas múltiplas de morte houve um incremento de $58 \%$ na mortalidade proporcional. Tal informação possibilitará o reconhecimento dessas como problemas de saúde pública, problemas estes que demandam programas específicos de prevenção e de assistência, além do maior investimento na área de saúde ambiental, visto que pacientes com doenças pulmonares obstrutivas crônicas constituem uma população suscetível a partículas aéreas urbanas. ${ }^{21}$ Enfatiza-se a necessidade de implantação de estratégias populacionais contra o tabagismo, por constituir esse hábito fator de risco para aquela patologia.

O enfoque das causas múltiplas de morte também aumentou a importância da cirrose hepática na mortalidade e tornou possível verificar a associação desta com a hepatite. Vários estudos epidemiológicos têm demonstrado a relação existente entre o consumo de álcool e a mortalidade por cirrose. Depois do alcoolismo, os vírus $\mathrm{B}$ e $\mathrm{C}$ da hepatite são os mais importantes fatores de risco para a cirrose. ${ }^{19}$ As mortes em idades produtivas são, geralmente, atribuídas ao maior consumo de álcool. ${ }^{22}$ Neste caso, estratégias populacionais, tais como a educação para a saúde enfocando mudanças no estilo de vida, de atitudes e de comportamentos tornam-se necessárias. Conforme Lessa, ${ }^{19}$ os benefícios atingiriam também outras doenças crônicas associadas ao consumo excessivo de álcool como as doenças cerebrovasculares, o diabetes secundário ao alcoolismo, a pancreatite e as doenças relacionadas à esfera psíquica. Outra importante estratégia de saúde seria a vacinação de hepatite $B$.

Observou-se que, com a análise da mortalidade sob o enfoque das causas múltiplas de morte, ocorre um aumento da proporção dos óbitos por algumas doenças crônicas não-transmissíveis. Nota-se também que as doenças do sangue e dos órgãos hematopoéticos, as doenças endócrinas, do metabolismo e nutricionais, bem como as doenças do aparelho geniturinário também merecem uma análise sob este enfoque. $\mathrm{O}$ número elevado de menções de doenças do aparelho respiratório pode ser explicado pelo fato de serem relativas a complicações ou afecções terminais de doenças.

Apesar de alguns autores ${ }^{3,5,11}$ utilizarem testes estatísticos para verificar a associação existente entre as causas de morte, não foi utilizado o recurso dessas técnicas, levando em consideração a premissa da independência nesses testes. Além disso, a abordagem descritiva permitiu evidenciar a magnitude de algumas associações de causas como problemas de saúde do adulto.

As políticas de saúde devem propiciar a promoção e proteção da saúde, evitando as mortes prematuras dos adultos, enfatizando o conhecimento e a prevenção dos fatores de risco passíveis de modificação, principalmente os relacionados aos hábitos de vida. Com a adoção de estilo de vida saudável, poderiam ser obtidos benefícios para as doenças cardiovasculares, mas, também, para o diabetes e algumas neoplasias, resultando em riscos menores de morte. ${ }^{19,23}$ Outro aspecto a ser considerado está relacionado ao ambiente, destacando-se o de trabalho. ${ }^{19}$

A análise das causas múltiplas de morte permite dimensionar a magnitude da mortalidade para várias doenças crônicas não-transmissíveis, demonstrando ser um instrumento alternativo de avaliação e planejamento das ações de saúde. Uma outra vantagem é a possibilidade de este enfoque tornar-se um importante incentivo para o preenchimento completo das DO. Acredita-se que os médicos responsáveis pelo preenchimento das declarações de óbito fiquem mais interessados quando constatarem que as DO podem fornecer novos conhecimentos quando, analisados através das causas múltiplas. Com a obtenção de maior informação, aumentarse-ia a utilidade das estatísticas de mortalidade para o planejamento dos serviços de saúde. 


\section{Agradecimentos}

Ao professor Mark Drew Crosland Guimarães pelas sugestões, ao professor Augusto Hasiak Santo pelas sugestões, cessão de referências bibliográficas e orientações na utilização do TCM e ao Celso Escobar Pinheiro pela orientação na utilização do TCM.

\section{Referências bibliográficas}

1. Laurenti R. Causas múltiplas de morte [tese de Doutorado]. São Paulo (SP): USP; 1973.

2. Santo AH. Causas múltiplas de morte: formas de apresentação e métodos de análise [tese de Doutorado]. São Paulo (SP): USP; 1988.

3. Israel RA, Rosenberg HM, Curtin, LR. Analytical potential of multiple causeof-death data. American Journal of Epidemiology 1986;124:161-179.

4. Westerling R. Small-area variation in multiple causes of death in Sweden: a comparison with underlying causes of death. International Journal of Epidemiology 1995; 24:552-558.

5. Guralnick L. Some problems in the use of multiple causes of death. Journal of Chronic Diseases 1966;19:979-990.

6. Organização Mundial da Saúde. Centro Colaborador da OMS para a Classificação de Doenças em Português. Classificação estatística internacional de doenças e problemas relativos a Saúde. v. 2. (Manual de instrução). $10^{\mathrm{a}}$ Revisão. São Paulo: EDUSP; 1994.

7. Tu EJ-C. Multiple cause-of-death analysis of hypertension-related mortality in New York State. Public Health Reports 1987;102:329-335.

8. Franco LJ, Mameri C, Pagliaro H, Iochida LC, Goldenberg P. Diabetes como causa básica ou associada de morte no Estado de São Paulo, Brazil, 1992. Revista de Saúde Pública 1998;32:237-245.

9. Melo MS, Lolio CA, Lucena MAF, Kirzner CF, Martins SM, Barros MNDS. Causas múltiplas de morte em diabéticos no município de Recife, 1987. Revista de Saúde Pública 1991;25:435-442.

10. Steenland K, Nowlin S, Ryan B, Adams S. Use of multiple-cause mortality data in epidemiologic analyses: US rate and proportion files developed by the National Institute for Occupational Safety and Health and the National Cancer Institute. American Journal of Epidemiology 1992;136:855-862.

11. Niobey FML. A análise de mortalidade infantil pós-neonatal sob a perspectiva das causas múltiplas, Rio de Janeiro, 1986/1987 [dissertação de Mestrado]. Rio de Janeiro (RJ): FIOCRUZ; 1994.

12. Santo AH, Pinheiro CE, Jordani MS. Causas básicas e associadas de morte por AIDS, Estado de São Paulo, Brasil, 1998. Revista de Saúde Pública 2000;34:581-588.

13. Lotufo PA. A mortalidade precoce por doenças crônicas nas capitais de regiões metropolitanas do Brasil [tese de Doutorado]. São Paulo (SP): USP; 1996.

14. Organização Mundial da Saúde. Centro Colaborador da OMS para a Classificação de Doenças em Português. Classificação estatística internacional de doenças e problemas relativos a Saúde. v.1. 10 ${ }^{\mathrm{a}}$ Revisão. São Paulo: EDUSP; 1993.

15. Santo AH, Pinheiro CE. Uso do microcomputador na seleção da causa básica de morte. Boletin de la Oficina Sanitaria Panamericana 1995;119:319327.

16. Ministério da Saúde. Secretaria Nacional de Ações Básicas de Saúde. Divisão Nacional de Epidemiologia. Lista brasileira para mortalidade. Brasília; 1980.

17. Santo AH, Pinheiro CE. Tabulador de causas múltiplas de morte. Revista Brasileira de Epidemiologia 1999;2:9097.

18. Population Reference Bureau. Cuadro de la población mundial. Datos y estimaciones demográficas de los 
países y regiones del mundo, 1996. Washington; 1996.

19. Lessa I. O adulto brasileiro e as doenças da modernidade; epidemiologia das doenças crônicas não-transmissíveis. São Paulo: HUCITEC; 1998.

20. Llanos G, Libman I. La diabetes en las Américas. Boletin de la Oficina Sanitaria Panamericana 1995;118:117.

21. Sunyer J, Schwartz J, Tobías A, Macfarlane D, Garcia J, Antó JM. Patients with chronic obstructive pulmonary disease are at increased risk of death associated with urban particle air pollution: a case-crossover analysis. American Journal of Epidemiology 2000;151:50-56.

22.Thun MJ, Peto R, Lopez AD, Monaco $\mathrm{JH}$, Henley J, Heath CW et al. Alcohol consumption and mortality among middle-aged and elderly adults. The New England Journal of Medicine 1997;337:1705-1715.

23. Johansson S-E, Sundquist J. Change in lifestyle factors and their influence on health status and all-cause mortality. International Journal of Epidemiology 1999;28:1073-1080. 